University of South Florida

DIGITAL COMMONS

Digital Commons @ University of

@ UNIVERSITY OF SOUTH FLORIDA

South Florida

$1-1-2011$

\title{
2011 Work Plan USF Sarasota-Manatee
}

USF

Follow this and additional works at: https://digitalcommons.usf.edu/usf_accountability_reports

\section{Scholar Commons Citation}

USF, "2011 Work Plan USF Sarasota-Manatee" (2011). USF Accountability Reports. 22.

https://digitalcommons.usf.edu/usf_accountability_reports/22

This Article is brought to you for free and open access by the USF Archives at Digital Commons @ University of South Florida. It has been accepted for inclusion in USF Accountability Reports by an authorized administrator of Digital Commons @ University of South Florida. For more information, please contact digitalcommons@usf.edu. 
2011 Update to the

University of South Florida

Sarasota-Manatee

Work Plan 
Note concerning data accuracy: The Office of the Board of Governors believes that the accuracy of the data it collects and reports is paramount to ensuring accountability in the State University System. Thus, the Board Office allows university resubmissions of some data to correct errors when they are discovered. This policy can lead to changes in historical data. 


\begin{tabular}{|c|c|c|c|c|c|c|c|}
\hline \multicolumn{8}{|c|}{ University of South Florida 2010 Annual Report } \\
\hline & & & \multicolumn{5}{|c|}{ USF Sarasota-Manatee } \\
\hline Enrollments & $\#$ & $\%$ & \multicolumn{3}{|c|}{ Degree Programs Offered (As of Spr. 10) } & \multicolumn{2}{|c|}{ Carnegie Classification } \\
\hline $\begin{array}{c}\text { TOTAL } \\
\text { (Fall 2009) }\end{array}$ & 1,784 & $100 \%$ & \multicolumn{2}{|l|}{ TOTAL } & 30 & $\begin{array}{c}\text { Undergraduate } \\
\text { Instructional Program: }\end{array}$ & \multirow{10}{*}{$\begin{array}{c}\text { SEPARATE CLASSIFICATION } \\
\text { PENDING }\end{array}$} \\
\hline Black & 110 & $6 \%$ & \multicolumn{2}{|c|}{ Baccalaureate } & 23 & Graduate Instructional & \\
\hline Hispanic & 152 & $9 \%$ & \multicolumn{2}{|c|}{ Master's \& Specialist's } & 7 & Program: & \\
\hline White & 1,408 & $79 \%$ & \multicolumn{2}{|c|}{ Research Doctorate } & 0 & Enrollment Profile: & \\
\hline Other & 114 & $6 \%$ & \multicolumn{2}{|c|}{ Professional Doctorate } & 0 & Undergraduate Profile: & \\
\hline Full-Time & 693 & $39 \%$ & \multirow{2}{*}{$\begin{array}{c}\text { Faculty } \\
\text { (Fall 2009) }\end{array}$} & \multirow{2}{*}{$\begin{array}{l}\text { Full- } \\
\text { Time }\end{array}$} & \multirow{2}{*}{$\begin{array}{l}\text { Part- } \\
\text { Time }\end{array}$} & Size and Setting: & \\
\hline Part-Time & 1,091 & $61 \%$ & & & & \multirow{2}{*}{ Basic: } & \\
\hline Undergraduate & 1,414 & $79 \%$ & TOTAL & 52 & 5 & & \\
\hline Graduate & 203 & $11 \%$ & Tenure/T. Track & 31 & 1 & \multirow{2}{*}{ Elective Classification: } & \\
\hline Unclassified & 167 & $9 \%$ & Other Faculty/Instr. & 21 & 4 & & \\
\hline
\end{tabular}

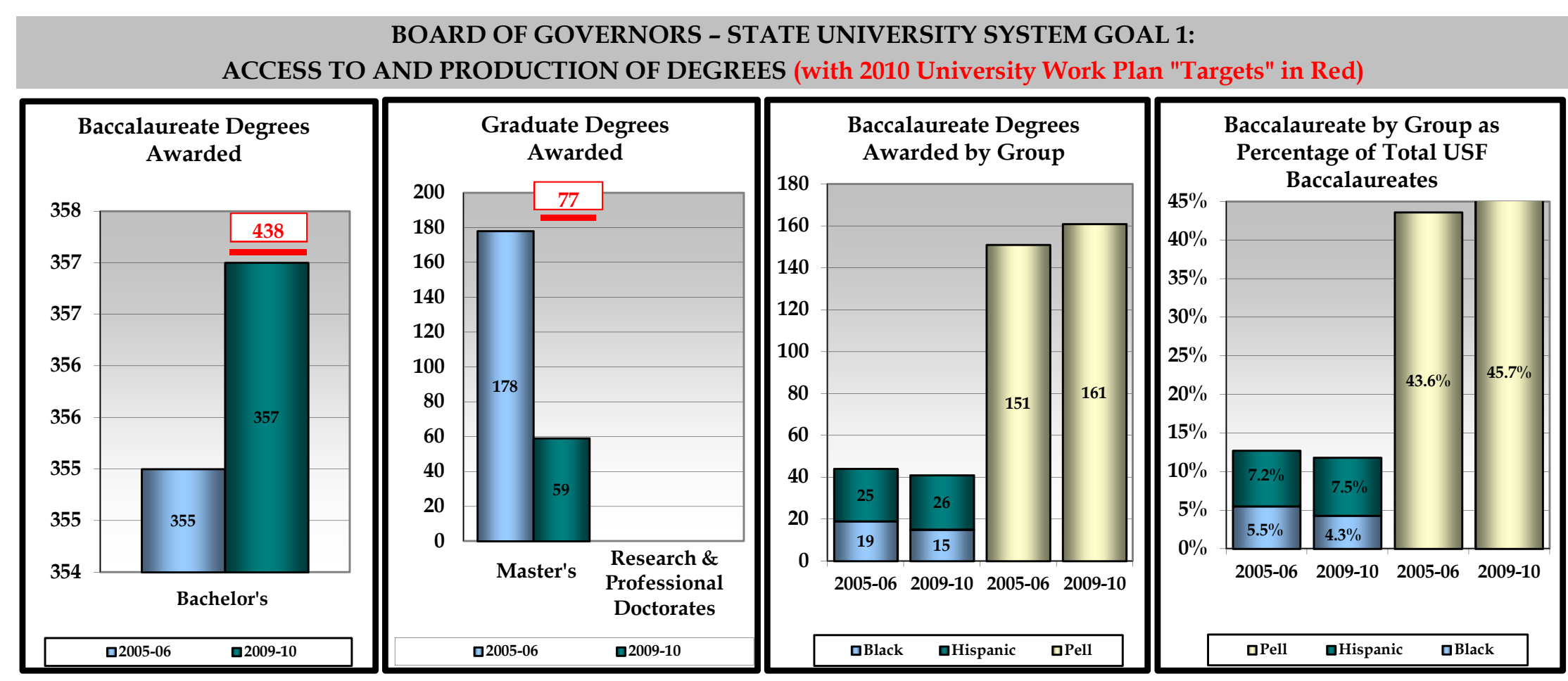

2012 - 2013 Projected Institutional Contributions in RED PRINT. 
BOARD OF GOVERNORS - STATE UNIVERSITY SYSTEM GOAL 2:

MEETING STATEWIDE PROFESSIONAL AND WORKFORCE NEEDS (with 2010 University Work Plan "Targets" in Red)

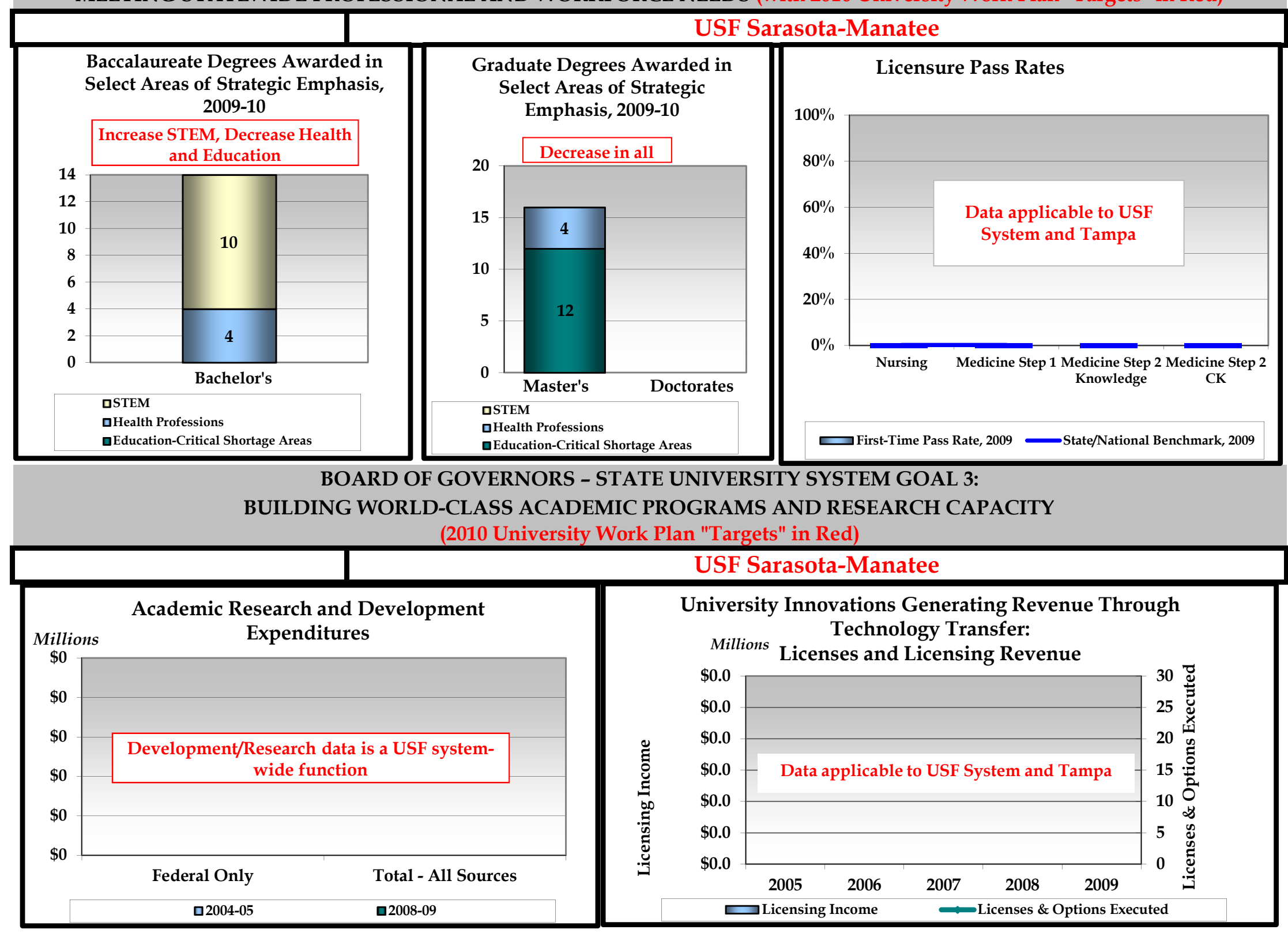

Projected Institutional Contributions in RED PRINT

(2012 - 2013 for TOTAL Degrees in Areas of Strategic Emphasis; 2012 for NCLEX; 2011 -2012 for R\&D, Licences, and Licensing Revenue). 


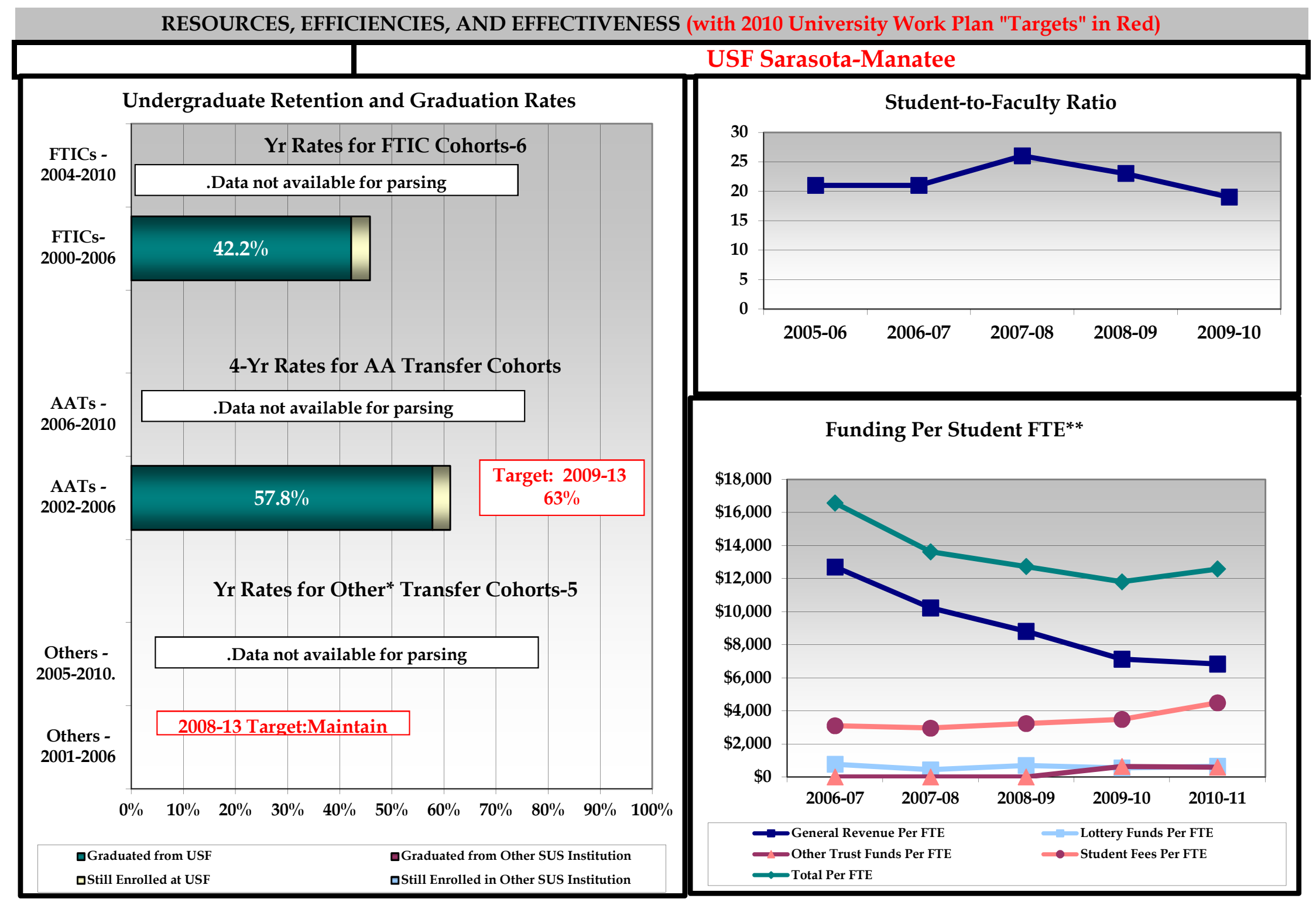

* The composition of "Other Transfer" cohorts may vary greatly by institution and by year.

** FTE for this metric uses the standard IPEDS definition of FTE, equal to 30 credit hours for undergraduates and 24 for graduates.

Graduation Rate from SAME Institution - Projected Institutional Contributions in RED PRINT. 


\section{Select Data Tables from the 2009-2010 Annual Report}

*Peer choices should be noted. In cases in which peer data are not available for a specific metric, but are available for a related metric, an institution might want to note such in the "Comparison with Peers" row.

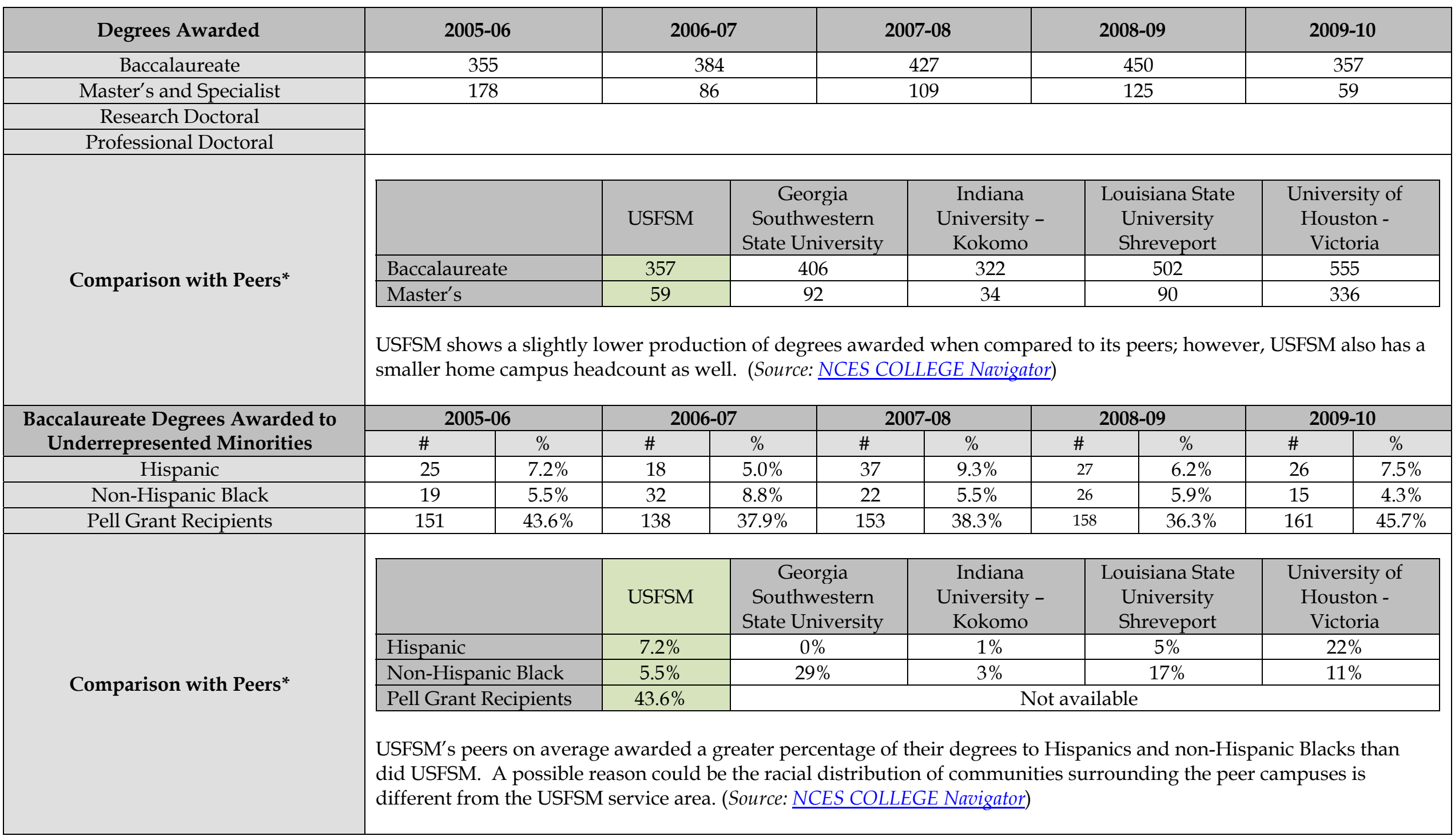




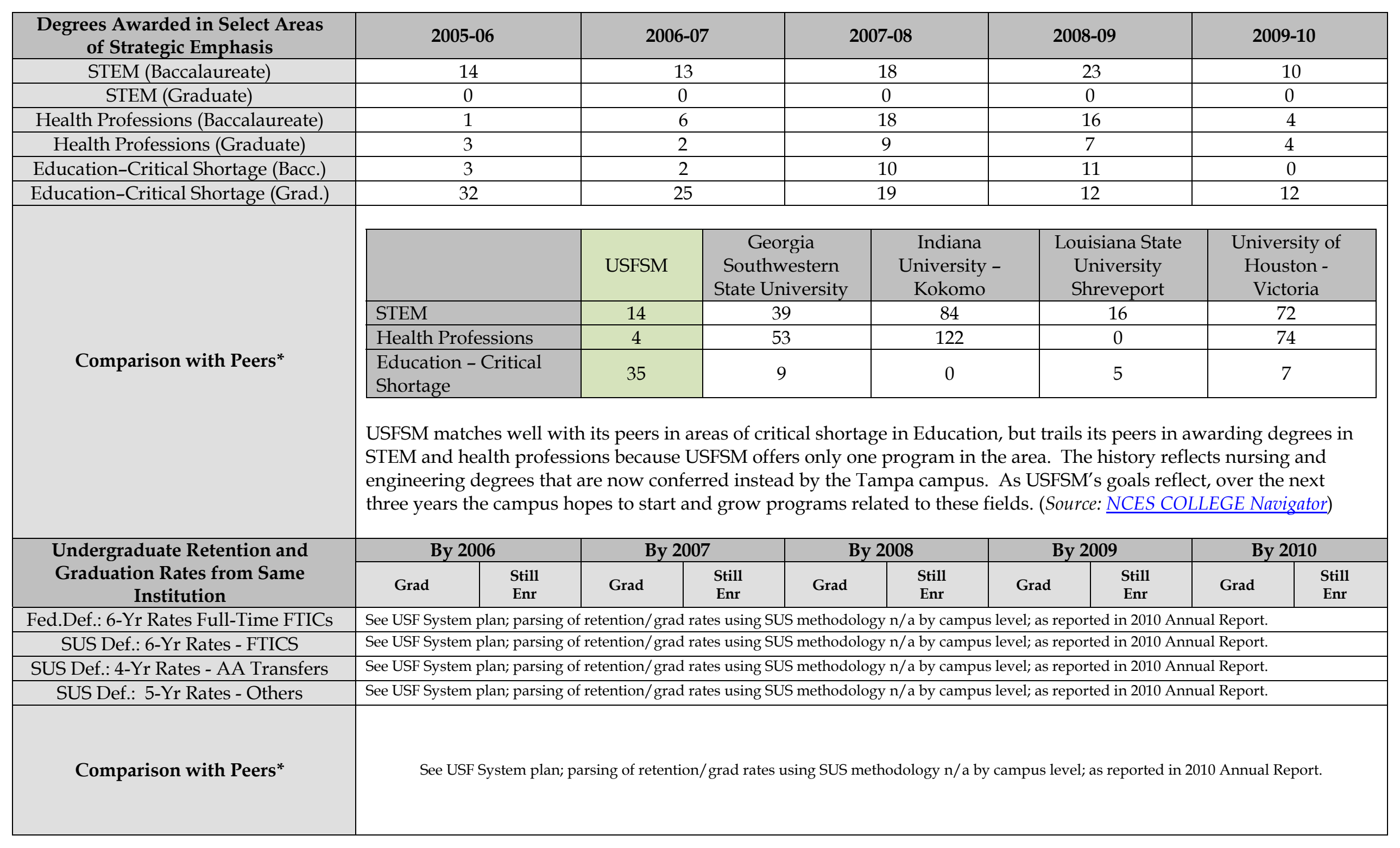




\begin{tabular}{|c|c|c|c|c|c|}
\hline Licensure Exam Pass Rates & Year 1 & Year 2 & Year 3 & Year 4 & Year 5 \\
\hline Nursing (2005-06 Through 2009-10) & $\mathrm{N} / \mathrm{A}$ & $\mathrm{N} / \mathrm{A}$ & $\mathrm{N} / \mathrm{A}$ & $\mathrm{N} / \mathrm{A}$ & $\mathrm{N} / \mathrm{A}$ \\
\hline Medicine - Step $1(2006$ - 2010) & $\mathrm{N} / \mathrm{A}$ & $\mathrm{N} / \mathrm{A}$ & $\mathrm{N} / \mathrm{A}$ & $\mathrm{N} / \mathrm{A}$ & $\mathrm{N} / \mathrm{A}$ \\
\hline $\begin{array}{l}\text { Medicine - Step } 2 \text { Clinical } \\
\text { Knowledge } \\
(2005-06 \text { Through 2009-10) }\end{array}$ & $\mathrm{N} / \mathrm{A}$ & $\mathrm{N} / \mathrm{A}$ & $\mathrm{N} / \mathrm{A}$ & $\mathrm{N} / \mathrm{A}$ & $\mathrm{N} / \mathrm{A}$ \\
\hline $\begin{array}{l}\text { Medicine - Step } 2 \text { Clinical Skills } \\
(2005-06 \text { Through 2009-10) }\end{array}$ & $\mathrm{N} / \mathrm{A}$ & $\mathrm{N} / \mathrm{A}$ & $\mathrm{N} / \mathrm{A}$ & $\mathrm{N} / \mathrm{A}$ & N/A \\
\hline Comparison with Peers* & \multicolumn{5}{|l|}{ Not applicable. } \\
\hline $\begin{array}{c}\text { Academic Research and } \\
\text { Development Expenditures }\end{array}$ & 2004-05 & $2005-06$ & 2006-07 & 2007-08 & 2008-09 \\
\hline Federal Only (Thousand \$) & $\mathrm{N} / \mathrm{A}$ & $\mathrm{N} / \mathrm{A}$ & $\mathrm{N} / \mathrm{A}$ & $\mathrm{N} / \mathrm{A}$ & N/A \\
\hline Total - All Sources (Thousand \$) & $\mathrm{N} / \mathrm{A}$ & $\mathrm{N} / \mathrm{A}$ & $\mathrm{N} / \mathrm{A}$ & $\mathrm{N} / \mathrm{A}$ & $\mathrm{N} / \mathrm{A}$ \\
\hline Comparison with Peers* & \multicolumn{5}{|l|}{ Not applicable. } \\
\hline Technology Transfer & 2005 & 2006 & 2007 & 2008 & 2009 \\
\hline Licenses \& Options Executed & $\mathrm{N} / \mathrm{A}$ & $\mathrm{N} / \mathrm{A}$ & $\mathrm{N} / \mathrm{A}$ & $\mathrm{N} / \mathrm{A}$ & $\mathrm{N} / \mathrm{A}$ \\
\hline Licensing Income & $\mathrm{N} / \mathrm{A}$ & $\mathrm{N} / \mathrm{A}$ & $\mathrm{N} / \mathrm{A}$ & $\mathrm{N} / \mathrm{A}$ & $\mathrm{N} / \mathrm{A}$ \\
\hline Comparison with Peers* & \multicolumn{5}{|l|}{ Not applicable. } \\
\hline $\begin{array}{l}\text { OTHER KEY OUTPUT OR } \\
\text { OUTCOME METRICS }\end{array}$ & 2005-06 & 2006-07 & 2007-08 & 2008-09 & 2009-10 \\
\hline & & & & & \\
\hline
\end{tabular}


Comparison with Peers*

Based on Review of Data Trends on Key Output or Outcome Metrics Identified Here and/or in Annual Report, Three (3) Areas of Concern/Areas Needing Improvement

(1) Separate accreditation in June 2011 will allow USFSM to offer new degree programs and courses that meet the needs of local employers. In alignment with the New Florida Initiative and USFSM's mission, the new degrees will be related to fields needed for regional and statewide development. To this end USFSM hopes to increase its degree production by targeting areas with strategic emphasis, such as science, arts, health, and technology.

(2)

(3) 


\section{UPDATES TO 2010 UNIVERSITY WORK PLAN}

[Please identify briefly any critical changes only to information provided in the 2010 University Work Plan that was not updated in the 2009-2010 Annual Report regarding the institution's strategic plan; institutional mission, vision, and strategic directions for the next five to ten years; current or aspirational peer institutions; windows of opportunity; or unique challenges.]

\section{CHANGES TO INSTITUTIONAL STRATEGIC PLAN}

USFSM STRATEGIC PLAN 2012-17: In June 2011, USFSM expects to be awarded separate regional accreditation from the Southern Association of Colleges and Schools. Though the campus will remain part of the USF System, the curricular autonomy will allow USF Sarasota-Manatee (USFSM) to develop new degree programs and courses, and to grow enrollment. In response to the changes occurring on the campus, Dr. Arthur Guilford, CEO of USFSM, recently charged a campus committee to develop a new strategic plan that will guide USFSM through the next five years. A new vision and mission is being developed, along with identifying the institution's specific goals and values. Initial discussions have been centered around (1) growing USFSM into a four-year degree institution and (2) developing programs based on their positive potential impact on the local community while also serving state and national educational needs, including a master's degree in Hotel and Restaurant Management, a School of Wellness and Longevity and programs in science, health, arts, and technology.

\section{SELECTED INSTITUTIONAL PEERS \& ASPIRANTS}

\section{Peers}

Georgia Southwestern State University

Indiana University Kokomo

Louisiana State University Shreveport

University of Houston Victoria

\section{Aspirant}

Auburn University at Montgomery

University of Houston at Clear Lake

University Of North Carolina at Asheville

The University of Texas of the Permian Basin

\section{WINDOWS OF OPPORTUNITY}

USFSM MOTE MARINE LABORATORY PARTNERSHIP: USFSM is working in partnership with Mote Marine Laboratory to bring the natural sciences to USFSM students. Potential benefits of the partnership include classroom laboratory space on the Mote campus and attracting Mote scientists into instructional roles so that they may share their expertise with the next generation of environmental stewards.

ON-LINE PEDAGOGY: In partnership with the Manatee and Sarasota County School Districts, USFSM will be training high school teachers in on-line pedagogy to prepare them for the new Florida legislation requiring that each student take an on-line course while enrolled in high school.

SARASOTA ARTS COMMUNITY: Sarasota is one of the cultural centers of Florida and rivals some of the biggest urban centers in the country. With dozens of performing arts venues, renowned opera, an orchestra, theater and ballet companies, numerous art museums, and a developing film industry, Sarasota offers a perfect learning environment for USF Sarasota-Manatee students to enrich their university education through the arts. The faculty in both the College of Business and the College of Education are working closely with the Sarasota arts community to infuse the arts into the MBA program and the teacher education program.

USF SYSTEM: As one of the four member institutions within the USF System, USFSM benefits from the efficiency of shared resources, the collaboration with the other member institutions, and a unified brand that yields identity and impact. 


\section{UNIQUE CHALLENGES}

FINANCIAL RESOURCES: USFSM complied with the Florida state law for separate regional accreditation in spite of a $46 \%$ decrease in general revenue funding per student FTE over the 2006-07 level.

STATE COLLEGE SYSTEM AND PRIVATE HIGHER EDUCATION: USFSM is experiencing increased competition and duplication of programs/services from state colleges and the for-profit private higher education market.

RECODING OF DEGREES: USF Sarasota-Manatee began the process of seeking separate accreditation in 2009. In Fall 2009, USF Tampa mandated that any USF Sarasota-Manatee student in degree programs hosted by USF Tampa or not offered completely by USF Sarasota-Manatee be removed from USF Sarasota-Manatee Home Campus enrollment numbers. As a result of this mandate, the number of degrees awarded by USF Sarasota-Manatee dropped significantly although the students still graduated from USF Tampa. 
CAVP Academic Coordination Project (List degree programs recommended for new collaborative or joint delivery model or other corrective action, as well as any degree programs recommended for continuation but for which university and Board staff have not reached agreement on the sufficiency of the rationale.)

\begin{tabular}{|c|c|c|c|c|c|}
\hline $\begin{array}{c}\text { Program } \\
\text { Level }\end{array}$ & $\begin{array}{l}\text { 6-Digit } \\
\text { CIP } \\
\text { Code }\end{array}$ & \multicolumn{2}{|c|}{ Program Title } & $\begin{array}{c}\text { Category (i.e., } \\
\text { Collaborative Model, } \\
\text { Corrective Action, or } \\
\text { Proposed Continuation) }\end{array}$ & Proposed Action \\
\hline \multicolumn{6}{|c|}{$\begin{array}{l}\text { New Academic Degree Program Proposals - Next Three Years (Program development goals need } \\
\text { to align with the institutional strategic plan and System priorities.) }\end{array}$} \\
\hline \multicolumn{2}{|c|}{$\begin{array}{l}\text { Proposed Date of } \\
\text { Submission to } \\
\text { University Board of } \\
\text { Trustees }\end{array}$} & $\begin{array}{c}\text { Program } \\
\text { Level }\end{array}$ & $\begin{array}{l}\text { 6-Digit } \\
\text { CIP Code }\end{array}$ & Program Title & $\begin{array}{c}\text { Comments } \\
\text { (Including Proposed } \\
\text { Implementation Date) }\end{array}$ \\
\hline \multicolumn{2}{|c|}{ TBD } & M & 13.1305 & Sec. Ed English Education & Fall 2012 \\
\hline \multicolumn{2}{|c|}{ TBD } & $\mathrm{M}$ & 13.1201 & Adult Education & Fall 2012 \\
\hline
\end{tabular}




\section{Enrollment Planning}

Please explain briefly any planned changes in enrollment patterns in the next five years, with rationale (e.g., more emphasis on enrolling FCS AA transfers; enrollment of more out-of-state students; enrollment of more FTICs as the institution builds out a more residential experience for undergraduates; maintain undergraduate enrollment with more growth at graduate level to align with institutional mission; plan to maintain current enrollment with more emphasis on improving graduation rates; etc.).

USF Sarasota-Manatee has plans to implement lower-level curriculum beginning in fall 2012. In addition, the campus has plans to add additional degree programs at both bachelor and master's levels that meet local and statewide needs. USFSM has been strategic in its selection of new programs, targeting areas that will best utilize resources while maximizing enrollment growth.

1. Annual FTE enrollment plans by level, site, and residency for tuition purposes in the format provided in the template on the next pages.

2. These are only to include fundable FTE enrollments. So, for example, out-of-state profile admits should not be included in the out-of-state data.

3. Remember that Pharm.D., Law, and other Professional Doctorates (per the recently changed IPEDS definitions) should be counted as Grad II enrollments.

4. An explanation of over-enrollment is required for any level in which the 2010-11 funded enrollment plan lagged actual 2010-11 enrollment by more than 5\% (Section 1011.90, F.S.). 


\begin{tabular}{|c|c|c|c|c|c|c|c|c|}
\hline \multicolumn{9}{|c|}{$\begin{array}{l}\text { Enrollment Plan Proposal - All State-Fundable FTE Enrollments } \\
\text { (Except Medical/Dental/Veterinary Enrollments) }\end{array}$} \\
\hline $\begin{array}{l}\text { For entire } \\
\text { institution }\end{array}$ & Funded & Estimated & Funded & Estimated & Estimated & Estimated & Estimated & \multirow{2}{*}{$\begin{array}{c}\text { 5-Year } \\
\text { Projected } \\
\text { Average } \\
\text { Annual } \\
\text { Growth } \\
\text { Rate } \\
\end{array}$} \\
\hline FTE & $2010-11$ & $2010-11$ & 2011-12 & 2011-12 & $2012-13$ & 2014-15 & 2016-17 & \\
\hline $\begin{array}{c}\text { FL } \\
\text { Resident } \\
\text { Lower }\end{array}$ & 0 & 64 & 0 & 69 & 97 & 252 & 472 & $117 \%$ \\
\hline $\begin{array}{c}\text { FL } \\
\text { Resident } \\
\text { Upper }\end{array}$ & 798 & 983 & 798 & 1033 & 1080 & 1179 & 1287 & $5 \%$ \\
\hline $\begin{array}{c}\text { FL } \\
\text { Resident } \\
\text { Grad I }\end{array}$ & 182 & 122 & 182 & 123 & 141 & 153 & 168 & $7.3 \%$ \\
\hline $\begin{array}{c}\text { FL } \\
\text { Resident } \\
\text { Grad II }\end{array}$ & 0 & 2 & 0 & 0 & 0 & 0 & 0 & 0 \\
\hline $\begin{array}{l}\text { Total FL } \\
\text { Resident }\end{array}$ & 980 & 1171 & 980 & 1225 & 1318 & 1584 & 1927 & $11.5 \%$ \\
\hline $\begin{array}{c}\text { Non-Res. } \\
\text { Lower }\end{array}$ & & 2 & & 0 & 0 & 0 & 0 & \\
\hline $\begin{array}{l}\text { Non-Res. } \\
\text { Upper }\end{array}$ & & 21 & & 16 & 16 & 18 & 20 & $5 \%$ \\
\hline $\begin{array}{c}\text { Non-Res. } \\
\text { Grad I }\end{array}$ & & 2 & & 3 & 3 & 4 & 4 & $6.7 \%$ \\
\hline $\begin{array}{c}\text { Non-Res. } \\
\text { Grad II }\end{array}$ & & 0 & & 0 & 0 & 0 & 0 & \\
\hline $\begin{array}{l}\text { Total Non- } \\
\text { Res. }\end{array}$ & 0 & 25 & 0 & 19 & 19 & 22 & 24 & $5.2 \%$ \\
\hline $\begin{array}{c}\text { Total } \\
\text { Lower }\end{array}$ & & 66 & & 69 & 97 & 252 & 472 & $117 \%$ \\
\hline $\begin{array}{c}\text { Total } \\
\text { Upper }\end{array}$ & & 1004 & & 1049 & 1096 & 1197 & 1307 & $4.9 \%$ \\
\hline $\begin{array}{c}\text { Total Grad } \\
\text { I }\end{array}$ & & 124 & & 126 & 144 & 157 & 172 & $7.3 \%$ \\
\hline $\begin{array}{l}\text { Total Grad } \\
\text { II }\end{array}$ & & 2 & & 0 & 0 & 0 & 0 & $0 \%$ \\
\hline Total FTE & 980 & 1196 & 980 & 1244 & 1337 & 1606 & 1951 & $11 \%$ \\
\hline
\end{tabular}




\begin{tabular}{|c|c|c|c|c|c|c|c|c|}
\hline \multicolumn{9}{|c|}{ Enrollment Plan Proposal - Medical/Dental/Veterinary State-Fundable Enrollments } \\
\hline $\begin{array}{l}\text { For entire } \\
\text { institution }\end{array}$ & Funded & Estimated & Funded & Estimated & Estimated & Estimated & Estimated & \multirow{2}{*}{$\begin{array}{c}\text { 5-Year } \\
\text { Projected } \\
\text { Average } \\
\text { Annual } \\
\text { Growth } \\
\text { Rate }\end{array}$} \\
\hline Headcount & 2010-11 & 2010-11 & 2011-12 & 2011-12 & 2012-13 & 2014-15 & 2016-17 & \\
\hline $\begin{array}{c}\text { FL Resident } \\
\text { Medical } \\
\text { Headcount }\end{array}$ & & & & & & & & \\
\hline $\begin{array}{c}\text { Non-Res. } \\
\text { Medical } \\
\text { Headcount }\end{array}$ & & & & & & & & \\
\hline \multicolumn{9}{|c|}{$\begin{array}{l}\text { Total Medical } \\
\text { Headcount }\end{array}$} \\
\hline \multicolumn{9}{|l|}{$\begin{array}{l}\text { FL Resident } \\
\text { Dentistry } \\
\text { Headcount }\end{array}$} \\
\hline \multicolumn{9}{|l|}{$\begin{array}{l}\text { Non-Res. } \\
\text { Dentistry } \\
\text { Headcount }\end{array}$} \\
\hline \multicolumn{9}{|l|}{$\begin{array}{c}\text { Total } \\
\text { Dentistry } \\
\text { Headcount }\end{array}$} \\
\hline \multicolumn{9}{|l|}{$\begin{array}{c}\text { FL Resident } \\
\text { Veterinary } \\
\text { Headcount }\end{array}$} \\
\hline \multicolumn{9}{|l|}{$\begin{array}{l}\text { Non-Res. } \\
\text { Veterinary } \\
\text { Headcount }\end{array}$} \\
\hline $\begin{array}{c}\text { Total } \\
\text { Veterinary } \\
\text { Headcount }\end{array}$ & & & & & & & & \\
\hline
\end{tabular}

[This medical headcount is MD-only, not all HSC enrollments.] 
For each distinct physical location (main, branch, site, regional campus) that has or is planned to have more than 150 FTE State-fundable enrollments

SITE: USF Sarasota-Manatee

\begin{tabular}{|c|c|c|c|c|c|c|}
\hline & Estimated & Estimated & Estimated & Estimated & Estimated & \multirow{2}{*}{$\begin{array}{c}\text { 5-Year } \\
\text { Projected } \\
\text { Average } \\
\text { Annual } \\
\text { Growth Rate }\end{array}$} \\
\hline FTE & 2010-11 & 2011-12 & 2012-13 & 2014-15 & 2016-17 & \\
\hline Lower & 66 & 69 & 97 & 252 & 472 & $117 \%$ \\
\hline Upper & 1004 & 1049 & 1096 & 1197 & 1307 & $4.9 \%$ \\
\hline Grad I & 125 & 126 & 144 & 157 & 172 & $7.3 \%$ \\
\hline Grad II & 0 & 0 & 0 & 0 & 0 & $0 \%$ \\
\hline Total & 1196 & 1244 & 1337 & 1606 & 1951 & $11 \%$ \\
\hline \multicolumn{7}{|l|}{ SITE: } \\
\hline & Estimated & Estimated & Estimated & Estimated & Estimated & \multirow{2}{*}{$\begin{array}{c}\text { 5-Year } \\
\text { Projected } \\
\text { Average } \\
\text { Annual } \\
\text { Growth Rate }\end{array}$} \\
\hline FTE & 2010-11 & 2011-12 & 2012-13 & 2014-15 & 2016-17 & \\
\hline \multicolumn{7}{|l|}{ Lower } \\
\hline \multicolumn{7}{|l|}{ Upper } \\
\hline \multicolumn{7}{|l|}{ Grad I } \\
\hline \multicolumn{7}{|l|}{ Grad II } \\
\hline \multicolumn{7}{|l|}{ Total } \\
\hline \multicolumn{7}{|l|}{ SITE: } \\
\hline & Estimated & Estimated & Estimated & Estimated & Estimated & \multirow{2}{*}{$\begin{array}{c}\text { 5-Year } \\
\text { Projected } \\
\text { Average } \\
\text { Annual } \\
\text { Growth Rate }\end{array}$} \\
\hline FTE & 2010-11 & 2011-12 & 2012-13 & 2014-15 & 2016-17 & \\
\hline \multicolumn{7}{|l|}{ Lower } \\
\hline \multicolumn{7}{|l|}{ Upper } \\
\hline \multicolumn{7}{|l|}{ Grad I } \\
\hline \multicolumn{7}{|l|}{ Grad II } \\
\hline Total & & & & & & \\
\hline
\end{tabular}


For the sum of the remaining physical locations with fewer than 150 current or planned State-fundable FTE enrollments.

SITE: REMAINING PHYSICAL LOCATIONS University of South Florida Sarasota-Manatee @ North Port

\begin{tabular}{|c|c|c|c|c|c|c|}
\hline & Estimated & Estimated & Estimated & Estimated & Estimated & $\begin{array}{c}\text { 5-Year } \\
\text { Projected } \\
\text { Average } \\
\text { Annual } \\
\text { Growth Rate }\end{array}$ \\
\hline FTE & $\mathbf{2 0 1 0 - 1 1}$ & $\mathbf{2 0 1 1 - 1 2}$ & $\mathbf{2 0 1 2 - 1 3}$ & $\mathbf{2 0 1 4 - 1 5}$ & $\mathbf{2 0 1 6 - 1 7}$ & 0 \\
\hline Upper & 0 & 0 & 0 & 0 & 0 & $5 \%$ \\
\hline Grad I & 76 & 79 & 83 & 90 & 99 & $4 \%$ \\
\hline Grad II & 4 & 5 & 5 & 6 & 6 & 0 \\
\hline Total & 0 & 0 & 0 & 0 & 0 & $5 \%$ \\
\hline
\end{tabular}

For the sum of current or planned State-fundable FTE enrollments not served at a physical location.

SITE: VIRTUAL INSTRUCTION / DISTANCE LEARNING

\begin{tabular}{|c|c|c|c|c|c|c|}
\hline & Estimated & Estimated & Estimated & Estimated & Estimated & $\begin{array}{c}\text { 5-Year } \\
\text { Projected } \\
\text { Average } \\
\text { Annual } \\
\text { Growth Rate }\end{array}$ \\
\hline FTE & $\mathbf{2 0 1 0 - 1 1}$ & $\mathbf{2 0 1 1 - 1 2}$ & $\mathbf{2 0 1 2 - 1 3}$ & $\mathbf{2 0 1 4 - 1 5}$ & $\mathbf{2 0 1 6 - 1 7}$ & $1.5 \%$ \\
\hline Lower & 25 & 26 & 26 & 27 & 28 & $2.1 \%$ \\
\hline Upper & 449 & 458 & 467 & 486 & 506 & $2.6 \%$ \\
\hline Grad I & 23 & 23 & 24 & 25 & 26 & 0 \\
\hline Total II & 0 & 0 & 0 & 0 & 0 & $2.1 \%$ \\
\hline
\end{tabular}


Primary Institutional Goals/Metrics for the Next One to Three Years (In the context of the institutional strategic plan and vision, as well as System priorities, present three (3) to five (5) goals on which university effort will be focused in the next one to three years. Describe each goal, including whether the goal is new or continuing, the strategies for achieving that goal, the timeline and metrics by which success will be measured, expected outcomes, and assumptions, including financial, upon which the projected outcomes are predicated.) Each university is asked to include one goal associated with improved baccalaureate retention and graduation (e.g., improved first-year retention; reduce attainment gaps for underrepresented groups; improve graduation rates for AA transfers; etc.).

\begin{tabular}{|c|c|c|c|c|c|c|c|c|c|}
\hline \multicolumn{3}{|c|}{$\begin{array}{c}\text { Institutional Goal } \\
\text { [Indicate whether NEW or CONTINUING] }\end{array}$} & \multicolumn{3}{|c|}{ Implementation Strategies } & \multicolumn{4}{|c|}{ Metric(s)/Timeline/Expected Outcomes } \\
\hline \multicolumn{3}{|c|}{$\begin{array}{l}\text { \#1 (Required) - IMPROVE } \\
\text { BACCALAUREATE RETENTION AND } \\
\text { GRADUATION - (CONTINUING) }\end{array}$} & \multicolumn{3}{|c|}{$\begin{array}{l}\text { Purchased Hobson's, a web-based early } \\
\text { alert and student retention CRM } \\
\text { technology solution. Campus advisors } \\
\text { and faculty will work together using the } \\
\text { system to pinpoint students at-risk. Also, } \\
\text { implemented state-of-the-art degree } \\
\text { audit system, Degree Works, to promote } \\
\text { timely progression and to better } \\
\text { determine course scheduling needs. } \\
\text { Tuition differential will pay for the } \\
\text { additional undergraduate courses. }\end{array}$} & \multicolumn{4}{|c|}{$\begin{array}{l}\text { Metric: } \\
\text { Retention Rate } \\
\text { Timeline: } \\
\text { As of March 2011, USFSM began using the retention } \\
\text { module to manage communications and conduct } \\
\text { pilot testing with faculty. Information collected } \\
\text { from faculty will be tied to students within a } \\
\text { comprehensive database. } \\
\text { Expected Outcome: } \\
\text { By 2014-15, a } 2 \% \text { increase in retention is expected. }\end{array}$} \\
\hline \multicolumn{4}{|c|}{ Proposed Funding Source: $2011-12$} & \multicolumn{6}{|c|}{ Proposed Funding Source: $2012-13$} \\
\hline $\begin{array}{l}\text { State/ Tuition } \\
\text { Revenue (est.) }\end{array}$ & $\begin{array}{c}\text { Other } \\
\text { (Identify } \\
\text { Revenue } \\
\text { Source - e.g., } \\
\text { Private) }\end{array}$ & $\begin{array}{l}\text { Undergrad. } \\
\text { Tuition } \\
\text { Differential } \\
\text { Revenue } \\
\text { (est.) }\end{array}$ & $\begin{array}{l}\text { Total from } \\
\text { 2011-12 }\end{array}$ & $\begin{array}{l}\text { Undergrad. } \\
\text { Tuition } \\
\text { Differential } \\
\text { Revenue } \\
\text { (est.) }\end{array}$ & $\begin{array}{l}\text { Legislative } \\
\text { Budget } \\
\text { Request } \\
\text { (State Funds) }\end{array}$ & $\begin{array}{l}\text { State/ } \\
\text { Tuition } \\
\text { Revenue } \\
\text { (est.) }\end{array}$ & $\begin{array}{c}\text { Other } \\
\text { (Identify } \\
\text { Revenue } \\
\text { Source - } \\
\text { e.g., } \\
\text { Private) } \\
\end{array}$ & $\begin{array}{l}\text { Total from } \\
2012-13\end{array}$ & $\begin{array}{l}2012-13 \text { to } \\
2016-17 \\
\text { PECO/ } \\
\text { Courtelis } \\
\text { Request }\end{array}$ \\
\hline$\$ 45,000$ & $\$ 0$ & $\$ 376,838$ & $\$ 421,838$ & $\$ 430,606$ & $\$ 0$ & $\$ 45,000$ & $\$ 0$ & $\$ 475,606$ & $\$ 0$ \\
\hline
\end{tabular}




\begin{tabular}{|c|c|c|c|c|c|c|c|c|c|}
\hline \multicolumn{3}{|c|}{$\begin{array}{c}\text { Institutional Goal } \\
\text { [Indicate whether NEW or CONTINUING] }\end{array}$} & \multicolumn{3}{|c|}{ Implementation Strategies } & \multicolumn{4}{|c|}{ Expected Outcomes/Metric(s)/Timeline } \\
\hline \multicolumn{3}{|c|}{$\begin{array}{l}\text { \#2 (Required) - Provide freshman and } \\
\text { sophomore classes. (NEW) }\end{array}$} & \multicolumn{3}{|c|}{$\begin{array}{l}\text { Pending BOG and SACS approval, } \\
\text { program is slated to begin in fall } 2012 . \\
\text { Courses will be limited to simplify } \\
\text { instruction needs and program costs. }\end{array}$} & \multicolumn{4}{|c|}{$\begin{array}{l}\text { Metric: } \\
\text { 1-year retention rate } \\
\text { Timeline: } \\
\text { Fall } 2012 \text { to Fall } 2013 \\
\text { Expected Outcome: } \\
70 \% \text { 1-year retention rate of initial cohort. } \\
\text { Assumptions: } \\
\text { Board of Governors and SACS approval of lower } \\
\text { level course offerings. Approval of Legislative } \\
\text { Budget Request (LBR). }\end{array}$} \\
\hline \multicolumn{4}{|c|}{ Proposed Funding Source: $2011-12$} & \multicolumn{6}{|c|}{ Proposed Funding Source: $2012-13$} \\
\hline $\begin{array}{l}\text { State/ Tuition } \\
\text { Revenue (est.) }\end{array}$ & $\begin{array}{c}\text { Other } \\
\text { (Identify } \\
\text { Revenue } \\
\text { Source - e.g., } \\
\text { Private) }\end{array}$ & $\begin{array}{l}\text { Undergrad. } \\
\text { Tuition } \\
\text { Differential } \\
\text { Revenue } \\
\text { (est.) }\end{array}$ & $\begin{array}{l}\text { Total from } \\
2011-12\end{array}$ & $\begin{array}{l}\text { Undergrad } \\
\text { Tuition } \\
\text { Differential } \\
\text { Revenue } \\
\text { (est.) }\end{array}$ & $\begin{array}{l}\text { Legislative } \\
\text { Budget } \\
\text { Request } \\
\text { (State Funds) }\end{array}$ & $\begin{array}{l}\text { State/ } \\
\text { Tuition } \\
\text { Revenue } \\
\text { (est.) }\end{array}$ & $\begin{array}{c}\text { Other } \\
\text { (Identify } \\
\text { Revenue } \\
\text { Source - } \\
\text { e.g., } \\
\text { Private) } \\
\end{array}$ & $\begin{array}{l}\text { Total from } \\
2012-13\end{array}$ & $\begin{array}{l}2012-13 \text { to } \\
2016-17 \\
\text { PECO/ } \\
\text { Courtelis } \\
\text { Request }\end{array}$ \\
\hline$\$ 0$ & $\$ 0$ & $\$ 0$ & $\$ 0$ & $\$ 24,000$ & $\$ 856,412$ & $\$ 320,000$ & $\$ 0$ & $\$ 1,200,412$ & $\$ 0$ \\
\hline
\end{tabular}




\begin{tabular}{|c|c|c|}
\hline $\begin{array}{c}\text { Institutional Goal } \\
\text { [Indicate whether NEW or CONTINUING] }\end{array}$ & Implementation Strategies & Expected Outcomes/Metric(s)/Timeline \\
\hline $\begin{array}{l}\text { \#3 (Required) - developing programs based } \\
\text { on their positive potential impact on the } \\
\text { local community while also serving state } \\
\text { and national educational needs, including a } \\
\text { School of Wellness and Longevity and } \\
\text { programs in science, health, arts, and } \\
\text { technology. (NEW) }\end{array}$ & $\begin{array}{l}\text { (1) Start } 2^{\text {nd }} \text { bachelor degree programs in } \\
\text { communication sciences and nursing (in } \\
\text { partnership with USF Health), and a } \\
\text { master's level degree in Social Work (in } \\
\text { partnership with USF Tampa). (2) } \\
\text { Partner with Mote Marine Laboratory to } \\
\text { offer course work in the natural sciences. } \\
\text { (3) Seek national accreditation for the } \\
\text { Colleges of Business and Education. }\end{array}$ & $\begin{array}{l}\text { Metric: } \\
\text { (1)Increased degree production in state and local } \\
\text { critical needs areas/strategic emphasis areas. (2) } \\
\text { Successful launch of an Interdisciplinary Natural } \\
\text { Science degree. (3) Successful accreditation of two } \\
\text { professional colleges (Business and Education). } \\
\text { Timeline: } \\
\text { (1)2nd BA in nursing start Summer 2011; MSW start } \\
\text { Fall 2011; and 2nd BA in CSD start Spring 2012. } \\
\text { Review of degree production } 3 \text { years after start of } \\
\text { program. (2) Interdisciplinary Natural Science start } \\
\text { in Fall 2013. (3) Business - AACSB separate } \\
\text { accreditation - 2013/Education - NCATE separate } \\
\text { accreditation - 2015. } \\
\text { Expected Outcomes: } \\
\text { (1) Double the number of strategic emphasis } \\
\text { degrees awarded by 2015. (2) First natural science } \\
\text { courses offered in partnership with Mote Marine to } \\
\text { start in Fall 2013. (3) Successful separate } \\
\text { professional accreditation from AACSB and } \\
\text { NCATE. }\end{array}$ \\
\hline
\end{tabular}




\begin{tabular}{|c|c|c|c|c|c|c|c|c|c|}
\hline \multicolumn{4}{|c|}{ Proposed Funding Source: 2011-12 } & \multicolumn{6}{|c|}{ Proposed Funding Source: 2012-13 } \\
\hline $\begin{array}{l}\text { State/ Tuition } \\
\text { Revenue (est.) }\end{array}$ & $\begin{array}{c}\text { Other } \\
\text { (Identify } \\
\text { Revenue } \\
\text { Source - e.g., } \\
\text { Private) }\end{array}$ & $\begin{array}{l}\text { Undergrad } \\
\text { Tuition } \\
\text { Differential } \\
\text { Revenue } \\
\text { (est.) }\end{array}$ & $\begin{array}{l}\text { Total from } \\
\text { 2011-12 }\end{array}$ & $\begin{array}{l}\text { Undergrad } \\
\text { Tuition } \\
\text { Differential } \\
\text { Revenue } \\
\text { (est.) }\end{array}$ & $\begin{array}{c}\text { Legislative } \\
\text { Budget } \\
\text { Request } \\
\text { (State Funds) }\end{array}$ & $\begin{array}{l}\text { State/ } \\
\text { Tuition } \\
\text { Revenue } \\
\text { (est.) }\end{array}$ & $\begin{array}{c}\text { Other } \\
\text { (Identify } \\
\text { Revenue } \\
\text { Source - } \\
\text { e.g., } \\
\text { Private) } \\
\end{array}$ & $\begin{array}{l}\text { Total from } \\
\text { 2012-13 }\end{array}$ & $\begin{array}{c}2012-13 \text { to } \\
2016-17 \\
\text { PECO/ } \\
\text { Courtelis } \\
\text { Request }\end{array}$ \\
\hline$\$ 0$ & $\$ 250,000^{*}$ & $\$ 0$ & $\$ 250,000$ & $\$ 0$ & 0 & $\$ 0$ & $\$ 225,000^{*}$ & $\$ 225,000$ & $\$ 0$ \\
\hline
\end{tabular}

OPTIONAL: Universities may add one or two additional goals.

\begin{tabular}{|c|c|c|c|c|c|c|c|c|c|c|}
\hline \multicolumn{11}{|c|}{ SUMMARY OF PROPOSED FUNDING FOR PRIMARY GOALS } \\
\hline \multicolumn{5}{|c|}{ Proposed Funding Source: $2011-12$} & \multicolumn{6}{|c|}{ Proposed Funding Source: 2012-13 } \\
\hline Goal \# & $\begin{array}{c}\text { State/ } \\
\text { Tuition } \\
\text { Revenue } \\
\text { (est.) }\end{array}$ & $\begin{array}{c}\text { Other } \\
\text { (Identify } \\
\text { Revenue } \\
\text { Source - } \\
\text { e.g., } \\
\text { Private) }\end{array}$ & $\begin{array}{l}\text { Undergrad } \\
\text { Tuition } \\
\text { Differential } \\
\text { Revenue } \\
\text { (est.) }\end{array}$ & $\begin{array}{l}\text { Total from } \\
2011-12\end{array}$ & $\begin{array}{l}\text { Undergrad } \\
\text { Tuition } \\
\text { Differential } \\
\text { Revenue } \\
\text { (est.) }\end{array}$ & $\begin{array}{l}\text { Legislative } \\
\text { Budget } \\
\text { Request } \\
\text { (State } \\
\text { Funds) }\end{array}$ & $\begin{array}{c}\text { State/ } \\
\text { Tuition } \\
\text { Revenue } \\
\text { (est.) }\end{array}$ & $\begin{array}{c}\text { Other } \\
\text { (Identify } \\
\text { Revenue } \\
\text { Source - } \\
\text { e.g., } \\
\text { Private) }\end{array}$ & $\begin{array}{l}\text { Total } \\
\text { from } \\
\text { 2012-13 }\end{array}$ & $\begin{array}{c}2012-13 \text { to } \\
2016-17 \\
\text { PECO/ } \\
\text { Courtelis } \\
\text { Request }\end{array}$ \\
\hline 1 & $\$ 45,000$ & $\$ 0$ & $\$ 376,838$ & $\$ 421,838$ & $\$ 430,606$ & $\$ 0$ & $\$ 45,000$ & $\$ 0$ & $\$ 475,606$ & $\$ 0$ \\
\hline 2 & $\$ 0$ & $\$ 0$ & $\$ 0$ & $\$ 0$ & $\$ 24,000$ & $\$ 856,412$ & $\$ 320,000$ & $\$ 0$ & $\$ 1,200,412$ & $\$ 0$ \\
\hline 3 & $\$ 0$ & $\$ 250,000^{*}$ & $\$ 0$ & $\$ 250,000$ & $\$ 0$ & $\$ 0$ & $\$ 0$ & $\$ 225,000^{*}$ & $\$ 225,000$ & $\$ 0$ \\
\hline Total & $\$ 45,000$ & $\$ 250,000 *$ & $\$ 376,838$ & $\$ 671,838$ & $\$ 454,606$ & $\$ 856,412$ & $\$ 365,000$ & $\$ 225,000^{*}$ & $\$ 1,901,018$ & $\$ 0$ \\
\hline
\end{tabular}

*Private. 


\section{0 - 2011 Tuition Differential Update}

Provide the following information for the 2010-2011 Academic Year.

2010-2011 - 70\% Initiatives (List the initiatives

provided in the 2010-11 tuition differential request.)

Increase undergraduate course offerings.
University Update on Each Initiative

41 undergraduate courses were funded in fall semester 2010.

\section{Additional Detail, Where Applicable:}

Total Number of Faculty Hired or Retained (funded by tuition differential):

39 adjunct faculty and 1 phased retirement faculty, and 1 faculty overload were funded.

Total Number of Advisors Hired or Retained (funded by tuition differential):

0

41

Total Number of Course Sections Added or Saved (funded by tuition differential):

2010-2011 - 30\% Initiatives (list the initiatives provided in the 2010-11 tuition differential request)

Managed at USF System Level (See System Work Plan)

\section{Additional Information (estimates as of April 30, 2011):}

Unduplicated Count of Students Receiving at least

University Update on Each Initiative one Tuition Differential-Funded Award:

\$ Mean (per student receiving an award) of Tuition

Differential-Funded Awards:

137

$\$ 783$

\$ Minimum (per student receiving an award) of Tuition Differential-Funded Awards:

\$ Maximum (per student receiving an award) of Tuition Differential-Funded Awards:
$\$ 250$

$\$ 1000$ 


\section{Fall 2011 Request for an Increased Tuition Differential Fee}

\section{University: USF Sarasota-Manatee}

\section{Effective Date}

University Board of Trustees Approval Date:

June 8, 2011

Campus or Center Location

Campus or Center Location to which the Tuition

Differential fee will apply (If the entire university, Entire university. indicate as such):

Undergraduate Course(s)

Course(s). (If the tuition differential fee applies to all university undergraduate courses, indicate as such. If not, also provide a rationale for the differentiation among courses):

Current and Proposed Increase in the Tuition Differential Fee

Current Undergraduate Tuition Differential per credit hour:

Percentage tuition differential fee increase (calculated as a percentage of the sum of base tuition plus tuition differential):

$\$$ Increase in tuition differential per credit hour:

$\$$ Increase in tuition differential for 30 credit hours:

All university undergraduate courses.

Projected Differential Revenue Generated and Intended Uses

Incremental differential fee revenue generated in

2011-12 (projected):

Total differential fee revenue generated in 2011-12

(projected):

\begin{tabular}{|c|}
\hline \$ 207,165 \\
\hline$\$ 376,838$ \\
\hline
\end{tabular}




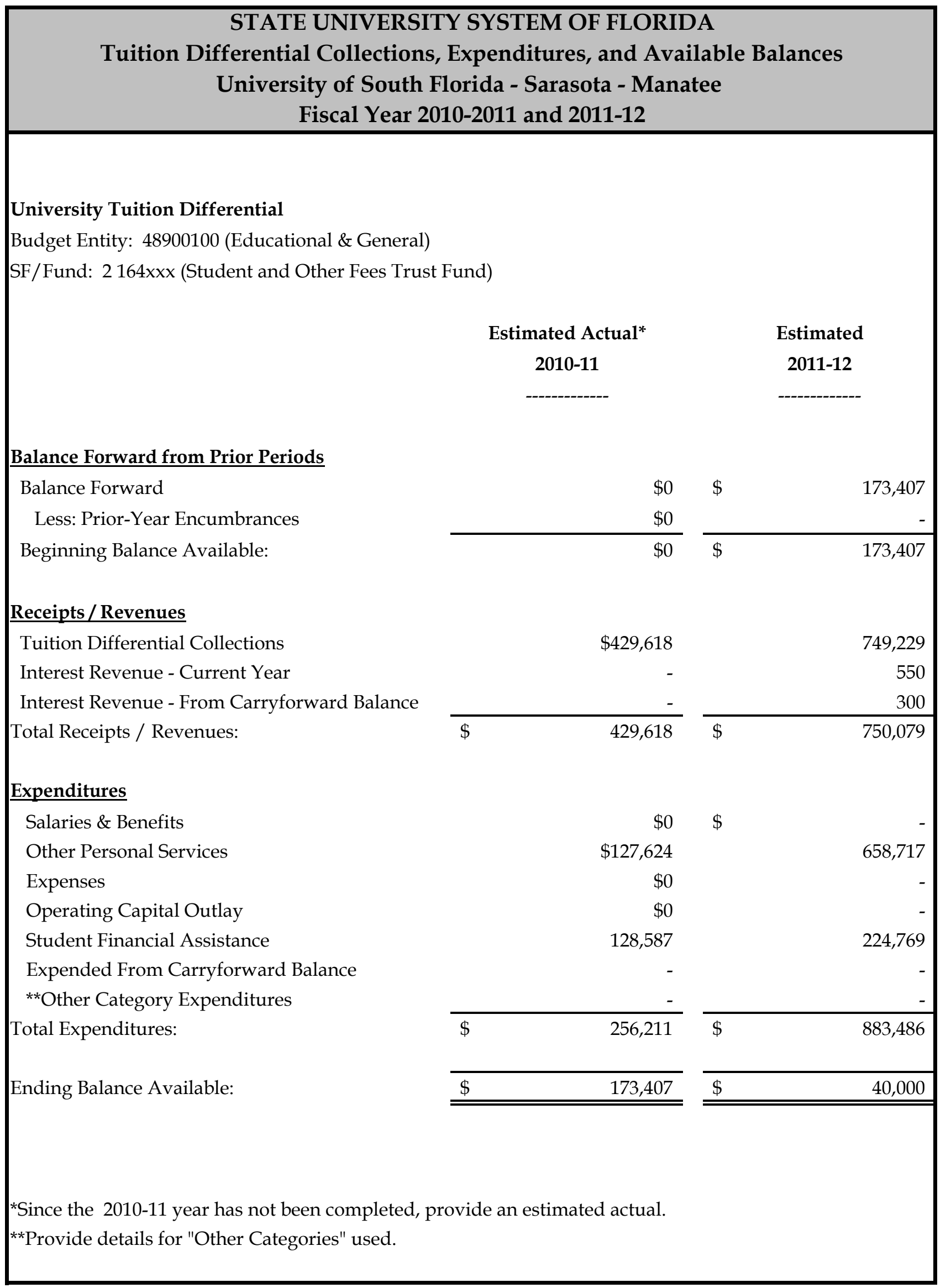




\section{State University System \\ Florida Board of Governors \\ Instructions for Completing the \\ Operating Budget (OB) Form I}

The OB Form I is designed to capture the data needed to align a university's work plan budget issue with the goals and objectives of the State University System (SUS) Strategic Plan and the New Florida Initiative.

Each university should submit one sequential priority list of all budget issues for the university. Any issues unique to a branch campus or a special unit (e.g., IFAS, health science center) should be incorporated into the single university priority list, even if the university decides to separate the base allocation into prorated amounts for each branch campus or special unit.

Keep all responses brief. All issues must have been identified in the 2011 University Work Plan submitted to the Board of Governors and must align with the goals and objectives of the SUS Strategic Plan and the New Florida Initiative. 


\section{State University System \\ Education and General \\ 2012-2013 Legislative Operating Budget Issue \\ Form I}

\begin{tabular}{|l|l|}
\hline University: & $\begin{array}{l}\text { University of South Florida Sarasota- } \\
\text { Manatee }\end{array}$ \\
\hline Work Plan Issue Title: & Lower-Level Curriculum \\
\hline Priority Number & $\mathbf{1}$ \\
\hline $\begin{array}{l}\text { Recurring Funds Requested: } \\
\text { Non-Recurring Funds Requested: }\end{array}$ & $\mathbf{\$ 8 5 6 , 4 1 2}$ \\
Total Funds Requested: & $\mathbf{\$ 0}$ \\
\hline
\end{tabular}

I. Description (Describe the service or program to be provided if this initiative is funded. Include whether this is a new or expanded service/program. If expanded, what has been accomplished with the current service/program?)

The University of South Florida Sarasota-Manatee proposes the offering of lowerlevel academic programs at the freshman and sophomore years for an entering cohort of 100 students beginning with Fall Semester 2012 to increase to 590 FTE by Fall Semester 2016.

The approval of this request will help the USF System meet the unmet and growing demand for lower-level academic programs at USF Sarasota-Manatee. Within Sarasota, Manatee, and DeSoto counties, employer demand for workers trained in high-skill, high-wage occupations continues to grow. Student enrollment in local institutions of higher education, including USF Sarasota-Manatee, also is growing. Local school districts and private, charter schools have expressed interest in partnering with USF Sarasota-Manatee in the development of dual enrollment programs.

Although this is a new service, USF Sarasota-Manatee tested the local demand by offering a few, select lower-level courses as a service to our entering transfer students who were lacking specific general education and prerequisite courses. In 2008-09 USF Sarasota-Manatee students took 269 credit hours in these courses. This number jumped nearly five times to 1,306 in 2009-10, and then doubled in 2010-11 to 2,640 credit hours.

II. Return on Investment (Describe the outcome(s) anticipated, dashboard indicator(s) to be improved, or return on investment. Be specific. For example, if this issue focuses on improving retention rates, indicate the current retention rate and the expected increase in the retention rate.)

This program will directly increase baccalaureate degree production within our region and will result in the many returns on investment that a well-educated work force brings to a local community. According to Pay Scale, Inc., the annual return on investment of earning a bachelor's degree at the University of South Florida is $10.9 \%$ 
(Source: http://www.payscale.com/education/average-cost-for-college-ROI) as compared to investing in stocks or bonds. Citizens with bachelor's degrees have a greater likelihood of achieving a higher socioeconomic status and income. They tend to pay substantially more taxes and lower burdens on government programs. They also have less likelihood of being jobless or incarcerated.

Within four years, USF Sarasota-Manatee's program will graduate the first cohort of students and grow each year after that. Students from USFSM's region will be more likely to remain in our region for their professional careers. Over 1,150 high school students leave Sarasota and Manatee counties annually to attend other state universities outside our region and are unlikely to return. USF Sarasota-Manatee's admissions office has 2,068 students in our database from high school graduating classes of 2011 and 2012, who expressed interest in attending USF SarasotaManatee as freshmen. Of these, nearly 300 are from the USF Sarasota-Manatee region. The local Economic Development Councils (EDC) and Chambers of Commerce are working to keep highly educated youth in our region, and USF Sarasota-Manatee offering lower-level course work will assist in meeting that mission. The cost of attendance at USF Sarasota-Manatee for a student living with his or her parents will be $\$ 5,000$ less annually than for students who leave the region to attend a state university. In addition, USF Sarasota-Manatee's student-faculty ratio is 15: 1, which is conducive to higher retention and graduation rates. When college freshman are in smaller university classes, they are much more likely to remain enrolled at the university and ultimately graduate with their bachelor's degrees.

III. Facilities (If this issue requires an expansion or construction of a facility and is on the Capital Improvement List complete the following table.): Not applicable

\begin{tabular}{|l|l|c|c|c|}
\hline & Facility Project Title & $\begin{array}{c}\text { Fiscal } \\
\text { Year }\end{array}$ & $\begin{array}{c}\text { Amount } \\
\text { Requested }\end{array}$ & $\begin{array}{c}\text { Priority } \\
\text { Number }\end{array}$ \\
\hline 1. & & & & \\
\hline 2. & & & & \\
\hline
\end{tabular}

Currently, USF Sarasota-Manatee's classrooms are at $60 \%$ capacity, providing ample classroom space for expansion into freshman and sophomore courses. 